\title{
Análise de padrões de transição ofensiva da Seleção Espanhola de Futebol na Copa do Mundo FIFA ${ }^{\circledR} 2010$
}

CDD. 20.ed. 796.073

796.33

http://dx.doi.org/10.1590/1807-55092015000100119
Rodrigo de Miranda Monteiro SANTOS*

Emerson Luciano MORAES*

Israel Teoldo da COSTA*
*Departamento de Educação Física, Universidade Federal de Viçosa.

\section{Resumo}

0 objetivo do artigo consiste em identificar os padrões de transição ofensiva (defesa-ataque) da seleção espanhola de futebol na Copa do Mundo FIFA 2010 através da análise da frequência das formas de aquisição/ recuperação da bola (FAR) e dos setores do campo onde se sucederam essas ações. A amostra do estudo integra 895 sequências ofensivas realizadas durante os sete jogos da seleção espanhola na competição. Para a coleta dos dados, utilizou-se a observação de vídeos de partidas televisionadas. Os dados foram registrados e quantificados utilizando-se planilhas de Excel 2007 for Windows ${ }^{\circledR}$. Realizou-se análise descritiva (frequência e percentual) para as variáveis "setor" e "FAR". 0 teste do qui-quadrado $\left(\chi^{2}\right)$ foi utilizado para comparar a distribuição dos casos entre os valores das variáveis analisadas com nivel de significância de $p<0,05$. Foram calculados os valores residuais padronizados (e) das FAR em função do setor do campo, e também do setor do campo de acordo com a FAR. Para o tratamento dos dados utilizou-se o "software" IBM SPSS versão 20. As variáveis "interceptação" e "fragmentos constantes do jogo" apresentaram frequência significativamente maior do que as outras FAR em todos os setores do campo, enquanto que a variável "interceptação" manifestou maior frequência nos setores defensivo $(e=2,73)$, médio defensivo $(e=5,51)$ e médio ofensivo $(e=$ $2,41)$ e a variável "desarme" teve frequência significativamente maior no setor médio defensivo $(e=4,85)$. Logo, conclui-se que houve maior ocorrência de fragmentos constantes do jogo no setor defensivo, de interceptações, no setor médio defensivo e médio ofensivo e de fragmentos constantes do jogo, no setor ofensivo, o que sugere que a seleção espanhola demonstra predisposição a evitar confrontos 1x1 no setor defensivo.

Palavras-chave: Futebol; Tática; Análise de jogo; Transição ofensiva.

\section{Introdução}

O emprego da análise de jogo no Futebol justifica-se pela importância da compreensáo dos padróes comportamentais relativos à dinâmica desta modalidade, bem como para subsidiar o planejamento dos processos de treino e ensino-aprendizagem, objetivando o máximo desempenho individual e coletivo $^{1-2}$. Dentre suas finalidades inclui-se a identificação das qualidades de uma equipe - visando sua manutenção - e no caso das com baixo desempenho - para que sejam desenvolvidas e atinjam melhor nível. Ao mesmo tempo, treinadores podem recorrer à análise de jogo para se defender dos pontos fortes de equipes adversárias, buscando contra-atacá-los, bem como explorar suas deficiências ${ }^{3-4}$. No entanto, certas características do comportamento das equipes, sobretudo durante a transição ofensiva, aparentam estar vinculadas à eficiência tática coletiva dentro da modalidade. Logo, pode-se afirmar que, considerando a transição como fundamental para a fase ofensiva, variáveis centrais para a avaliação da qualidade da transição defesa-ataque são os locais e as formas de recuperação da posse de bola ${ }^{5-6}$.

A recuperação da bola é definida por GARGANTA ${ }^{7}$ como o primeiro momento de posse de bola de uma equipe, coincidindo com o início da fase ofensiva de seu jogo. Portanto, ao se ter em conta a organização sistêmica do jogo de Futebol, compreende-se que a ação de recuperação da bola integra a transição defesaataque que, por sua vez, é entendido como o momento inicial da fase ofensiva ${ }^{8}$. Igualmente, de acordo com 
Pollard et al. ${ }^{9}$, o local do campo onde se sucedem as ações de recuperação da bola pode ser considerado como um indicador da aptidão de uma equipe para gerir de forma qualitativa a posse da bola. Assim, entendese que a localização e o tipo da ação são importantes parâmetros para observação e identificação de padróes comportamentais das equipes durante a fase ofensiva. Deste modo, para que seja possível identificar os locais onde ocorrem determinadas ações, faz-se necessário a delimitação de setores no campo ${ }^{10}$. Ainda, parâmetros que permitam identificar o tipo de ação para recuperação da bola são essenciais para a observação e análise das açôes durante a transição 5 . Portanto, foi considerada para este estudo a classificação proposta por GARGANTA", a qual o autor define como "formas de aquisição/recuperação da bola” (FAR).

Alguns dos estudos envolvendo a análise de padrôes comportamentais durante a transição defesa-ataque têm sido desenvolvidos com o intuito de explorar esta variável como um indicador de desempenho das equipes de futebol ${ }^{11-12}$. Outros têm se dedicado a investigar as açôes transicionais através da análise sequencial, visando identificar e estudar os métodos de jogo ofensivo no futebol ${ }^{13-14}$. Além disso, apesar da existência de investigações que

\section{Método}

\section{Amostra}

A amostra deste estudo foi composta por 895 sequências ofensivas realizadas durante os sete jogos disputados pela seleção espanhola de futebol durante a Copa do Mundo FIFA ${ }^{\oplus}$ 2010. A partir destas açóes, analisou-se a frequência de duas variáveis categóricas: a forma de aquisição/recuperação da bola (FAR) e o setor do campo onde esta ação ocorreu.

\section{Procedimento}

Para a coleta dos dados, utilizou-se a observação de imagens de vídeo a partir de partidas transmitidas por uma rede de televisão. As observaçôes dos vídeos dos jogos foram realizadas através do "software" de vídeo Windows Media Player ${ }^{\oplus}$. Os dados foram registrados e quantificados utilizando-se planilhas do "software" Excel 2007 for Windows ${ }^{\circledast}$. buscaram examinar aspectos relacionados ao modelo de jogo de equipes bem-sucedidas nos maiores torneios internacionais e domésticos (Copa do Mundo FIFA ${ }^{\circledast}$ e Liga Espanhola) ${ }^{15-16}$, estas utilizam variáveis cujos conceitos são dúbios e/ou pouco objetivos, o que limita a possibilidade de replicação dos experimentos e, como consequência, dificulta sua comparação com achados de outros trabalhos.

Desta forma, torna-se importante a identificação e análise das características específicas do modelo de jogo destas equipes - especialmente daquelas relacionadas ao comportamento ofensivo (pelo qual tais equipes são reconhecidas e admiradas pela mídia e torcedores ${ }^{17}$ ) - através da análise de variáveis que possuam definiçôes mais claras e objetivas, permitindo que pesquisadores e treinadores possam recorrer a estes dados para, respectivamente, desenvolver novas investigaçóes e desenvolver o processo de treino e de estudo dos adversários.

Assim, o objetivo deste estudo foi identificar os padróes de transição ofensiva (defesa-ataque) da seleção espanhola de futebol na Copa do Mundo FIFA $^{\oplus} 2010$ através da análise da frequência das formas de aquisição/recuperação da bola (FAR) e dos setores do campo onde ocorreram essas açóes.

Com o intuito de analisar a frequência das formas de aquisição/recuperação da bola (FAR) em cada um dos setores, utilizou-se o conceito de campograma proposto por GARGANTA ${ }^{7}$ e GréHAIGNE et al. ${ }^{10}$, que compreende quatro setores: setor defensivo (D), setor médio defensivo $(\mathrm{MD})$, setor médio ofensivo (MO) e setor ofensivo (O), como mostrado na FIGURA 1.

Para análise e registro das FAR, esta variável foi categorizada a partir dos conceitos apresentados por Garganta ${ }^{7}$ e Duprat e CATY ${ }^{5}$. Assim, foram consideradas para análise as seguintes formas de aquisição/recuperação da bola: 1) interceptação (jogador bloqueia o remate ou passe do portador da bola e recupera a posse); 2) desarme (oposição direta do jogador da equipe ao adversário portador da bola, com subsequente manutenção da posse de bola); 3) fragmentos constantes do jogo (tiros de meta, arremessos laterais, escanteios, pênaltis, tiros livres, impedimentos, defesas do goleiro e pontapés de saída); 4) erros do adversário (passe negativo). 


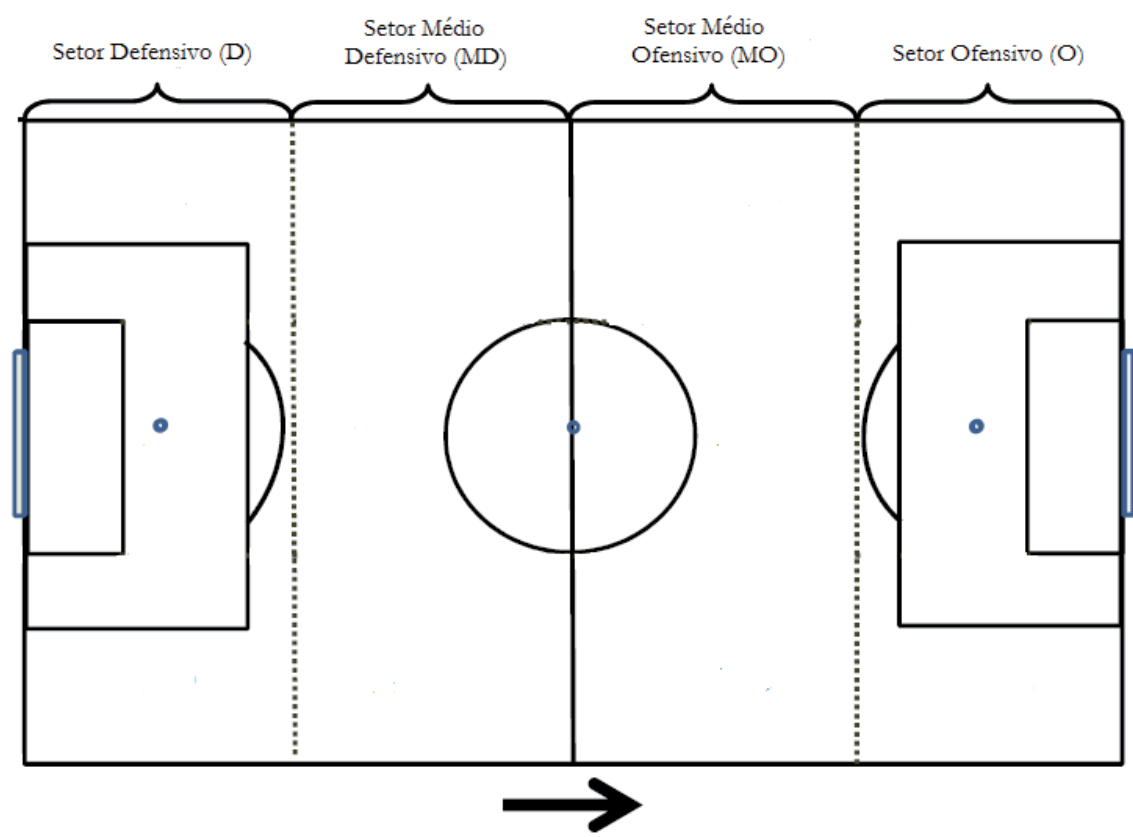

FIGURA 1 - Configuração espacial do campo de jogo de acordo com seus setores.

\section{Material}

A análise dos jogos foi realizada através de um computador portátil Acer, modelo Aspire 4738-6890.

\section{Análise estatística}

Realizou-se análise descritiva (frequência absoluta e relativa) para as variáveis "setor" e "forma de aquisição/recuperaçáo da bola" (FAR). O teste do qui-quadrado $\left(\chi^{2}\right)$ foi utilizado para comparação da distribuição dos casos entre os valores das variáveis categóricas analisadas, com distribuição teoricamente esperada ${ }^{18}$. O nível de significância adotado foi de $\mathrm{p}<0,05$. Para uma medida de magnitude padronizada do efeito observado ${ }^{19}$, calculou-se os tamanhos de efeito $(\omega)$ exercido pelas variáveis "setor" e "FAR" através da utilização da equação ${ }^{20}$ :

$$
\omega=\sqrt{\frac{\chi^{2}}{n}}
$$

Para a análise dos tamanhos de efeito, considerouse a classificação utilizada por $\operatorname{CoHEN}^{20}$, que os define como pequenos $(\omega=0,1$ ou $1 \%$ da variância total), médios ( $\omega=0,3$ ou $9 \%$ da variância total) e grandes ( $\omega=0,5$ ou $25 \%$ da variância total).

Foram calculados os valores dos resíduos padronizados (e) das FAR em funçáo do setor do campo e também do setor do campo em função das FAR, através de comparaçôes célula por célula, para que se pudesse determinar qual forma de aquisição/ recuperação da bola teve maior influência sobre o valor de $\chi^{2}$ e, por consequência, maior ocorrência em cada um dos setores, e também em qual setor cada uma destas formas ocorreu mais frequentemen$\mathrm{te}^{21-22}$. Os valores dos resíduos padronizados foram obtidos através da utilização da seguinte equaçãa ${ }^{23}$ :

$$
e=\frac{O-E}{\sqrt{E}}
$$

Foram consideradas influentes para o modelo, as células cujos valores de resíduo padronizado foram superiores a $2(\mathrm{e}>2)^{19}$.

Para o tratamento dos dados foi utilizado o "software" estatístico IBM SPSS (Statistical Package for Social Sciences) versão 20.

\section{Análise da fiabilidade}

Para o cálculo da fiabilidade adotou-se o método teste-reteste e se utilizou os valores do teste Kappa de Cohen para a descrição dos resultados. As observaçôes dos jogos foram realizadas por três observadores treinados. Foram reanalisadas 147 açóes de posse de bola, ou $16,42 \%$ da amostra, porcentagem superior àquela apontada como referência (10\%) pela literatura ${ }^{24}$. Os resultados de fiabilidade apresentaram valores de concordância inter-observadores entre 0,912 (ep $=0,032) \mathrm{e}$ 1 , e intra-observador entre $0,864(\mathrm{ep}=0,030)$ e 0,998 (ep = 0,002). Estes valores de fiabilidade são classificados por LANDis e $\mathrm{KoCH}^{25}$ como "quase perfeitos" (0,81 a 1$)$. 


\section{Resultados}

A TABELA 1 mostra a distribuição da frequência observada e os valores de qui-quadrado $\left(\chi^{2}\right)$ dos setores do campo no que diz respeito às formas de aquisição/recuperação da bola (FAR). É possível perceber a diferença na frequência das FAR dentro dos setores do campo, pois nota-se que o valor de $\chi^{2}$ mostrou-se significativo nos quatro setores analisados $(p<0,001)$, o que significa que em todos os setores, o valor da frequência de uma ou mais formas de aquisição/recuperação da bola foi significativo em relação aos das demais. No que diz respeito aos tamanhos de efeito $(\omega)$, percebe-se efeitos considerados grandes $(\omega>0,5)$ em todos os setores, o que confirma a discrepância entre as frequências já encontradas através do $\chi^{2}$.

TABELA 1 - Frequências (absolutas e relativas) observadas, valores de qui-quadrado $\left(\chi^{2}\right)$ e de tamanho de efeito das FAR em relação aos setores do campo.

${ }^{*} p<0,05$

${ }^{*} p<0,05$.

\begin{tabular}{lccccccc}
\hline Setor & Interceptação & Desarme & Fragmentos & Erros & $\chi^{2}$ & p & $\omega$ \\
\hline D & $62(33,7 \%)$ & $15(8,2 \%)$ & $82(44,6 \%)$ & $25(13,6 \%)$ & 64,21 & $<0,001^{*}$ & 0,590 \\
MD & $139(41,9 \%)$ & $50(15,1 \%)$ & $113(34 \%)$ & $30(9 \%)$ & 95,59 & $<0,001^{*}$ & 0,536 \\
MO & $110(40,3 \%)$ & $27(9,9 \%)$ & $103(37,7 \%)$ & $33(12,1 \%)$ & 86,37 & $<0,001^{*}$ & 0,562 \\
O & $39(36,8 \%)$ & $10(9,4 \%)$ & $49(46,2 \%)$ & $8(7,5 \%)$ & 48,18 & $<0,001^{*}$ & 0,674 \\
\hline
\end{tabular}

A TABELA 2 mostra a distribuição da frequência observada e os valores de qui-quadrado $\left(\chi^{2}\right)$ de cada uma das formas de aquisição/recuperação da bola (FAR) em relação aos setores do campo. Percebe-se a influência do setor nas açôes de recuperação da bola, pois todos os valores de $\chi^{2}$ mostraram-se significativos $(\mathrm{p} \leq 0,001)$ para todas as FAR. Aqui é possível observar uma variação maior dos valores de tamanhos de efeito $(\omega)$ para a ocorrência das FAR em cada setor, pois nota-se que apenas a variável "desarme" obteve um valor de tamanho de efeito que pode se considerar grande $(\omega=0,605)$, o que evidencia que a ocorrência desta ação deu-se com maior significância em 1 ou mais setores do campo em comparação aos demais.

TABELA 2 - Frequências (absolutas e relativas) observadas, valores de qui-quadrado $\left(\chi^{2}\right)$ e de tamanho de efeito dos setores do campo em relação às FAR.

\begin{tabular}{llccccccc} 
& FAR & $\mathbf{D}$ & MD & MO & O & $\chi^{\mathbf{2}}$ & $\mathbf{p}$ & $\boldsymbol{\omega}$ \\
\cline { 2 - 9 } & Interceptação & $62(17,7 \%)$ & $139(39,7 \%)$ & $110(31,4 \%)$ & $39(11,1 \%)$ & 70,41 & $<0,001^{*}$ & 0,448 \\
Desarme & $15(14,7 \%)$ & $50(49 \%)$ & $27(26,5 \%)$ & $10(9,8 \%)$ & 37,37 & $<0,001^{*}$ & 0,605 \\
Fragmentos & $82(23,6 \%)$ & $113(32,6 \%)$ & $103(29,7 \%)$ & $49(14,1 \%)$ & 26,67 & $<0,001^{*}$ & 0,282 \\
Erros & $25(26 \%)$ & $30(31,3 \%)$ & $33(34,4 \%)$ & $8(8,3 \%)$ & 15,58 & $0,001^{*}$ & 0,402 \\
\hline
\end{tabular}

A TABELA 3 apresenta os resíduos padronizados das FAR de acordo com cada setor do campo. Percebe-se que as variáveis "interceptaçáo" e "fragmentos constantes do jogo" apresentaram frequência significativamente maior do que as outras FAR em todos os setores do campo, mostrando que estas açóes ocorreram mais vezes em termos gerais quando comparadas às outras duas FAR.

TABELA 3 - Resíduos padronizados (e) das FAR em relação aos setores do campo.

\begin{tabular}{llcccc}
\cline { 2 - 5 } $\begin{array}{l}\text { *Valores residuais pa- } \\
\text { dronizados significativos } \\
(\mathrm{e}>2,0 \text { ou }<-2,0) .\end{array}$ & \multicolumn{5}{c}{ Resíduos padronizados $(\mathbf{e})$} \\
\cline { 2 - 6 } & Setor & Interceptaçáo & Desarme & Fragmentos & Erros \\
\cline { 2 - 5 } & MD & $2,36^{*}$ & $-4,57^{*}$ & $5,31^{*}$ & $-3,10^{*}$ \\
& MO & $6,15^{*}$ & $-3,62^{*}$ & $3,29^{*}$ & $-5,82^{*}$ \\
& $\mathrm{O}$ & $5,05^{*}$ & $-4,99^{*}$ & $4,21^{*}$ & $-4,27^{*}$ \\
& & $2,43^{*}$ & $-3,21^{*}$ & $4,37^{*}$ & $-3,59^{*}$ \\
\hline
\end{tabular}


A TABELA 4 mostra os resíduos padronizados dos setores do campo em relação às FAR. Aqui, nota-se a que a variável "interceptação" teve maior frequência nos setores defensivo $(e=2,73)$, médio defensivo (e $=5,51)$ e médio ofensivo $(e=2,41)$. A variável "desarme" teve frequência significativamente maior no setor médio defensivo $(e=4,85)$ em relação aos outros, assim como os "fragmentos constantes do jogo" que também apresentaram valor residual significativo no setor médio defensivo (e = 2,82). Já a variável "erros do adversário" não apresentou valor residual significativo em nenhum dos setores, apesar de ter obtido valor residual bruto superior $(e=1,84)$ no setor médio ofensivo.

TABELA 4 - Resíduos padronizados (e) dos setores do campo em relação às FAR.

\begin{tabular}{lcccc}
\hline \multicolumn{5}{c}{ Resíduos padronizados (e) } \\
\hline FAR & D & MD & MO & O \\
\hline Interceptação & $2,73^{*}$ & $5,51^{*}$ & $2,41^{*}$ & $-5,18^{*}$ \\
Desarme & $-2,08^{*}$ & $4,85^{*}$ & 0,30 & $-3,07^{*}$ \\
Fragmentos & $-0,51$ & $2,82^{*}$ & 1,74 & $-4,05^{*}$ \\
Erros & 0,20 & 1,22 & 1,84 & $-3,27^{*}$ \\
\hline
\end{tabular}

*Valores residuais padronizados significativos $(\mathrm{e}>2,0$ ou $<-2,0)$.

\section{Discussão}

O objetivo deste estudo foi identificar padróes de transição ofensiva (defesa-ataque) da seleção espanhola de futebol na Copa do Mundo FIFA ${ }^{\oplus}$ 2010 através da análise da frequência das formas de aquisição/recuperação da bola (FAR) e dos setores do campo onde ocorreram essas açóes.

Os achados do presente artigo indicaram que o setor médio defensivo foi o que proporcionou maior número de ações de recuperação da bola $(\mathrm{n}=332$; $\left.\chi^{2}=95,59\right)$, especialmente as de interceptação (e = 6,15), em comparaçáo aos outros setores. Assim, pode-se inferir que, a partir destes dados, a seleção espanhola demonstra tendência a recuperar a bola no setor médio defensivo através de bloqueios às tentativas de passe adversárias. O estudo de BARREIRA et al. ${ }^{26}$ sobre a seleção espanhola na UEFA ${ }^{\odot}$ Euro 2008 verificou padrôes de comportamento semelhantes durante a fase ofensiva, a partir das formas de recuperação da bola observadas. Esta tendência também se mostra em consonância com alguns dos princípios de funcionamento da organização defensiva desta equipe, que consistem na predisposição permanente à interceptação e na valorização do eixo longitudinal do campo, através da ampliação do número de linhas defensivas, com o objetivo de defender em profundidade ao invés de priorizar a largura destas linhas ${ }^{27-28}$.

Os resultados mostraram que o setor ofensivo foi o que maior efeito exerceu sobre a variabilidade das FAR $(\omega=0,674)$. Também neste setor, observou-se que a forma de recuperação da bola mais frequente foram os fragmentos constantes do jogo, sobre o que se pode deduzir que os adversários da equipe espanhola demonstram propensão a ceder a posse de bola através de lances de bola parada (escanteios, arremessos laterais ou tiros livres), ao invés de cedêla por outros meios (desarme, interceptação, erros). A partir disso, infere-se que ao optarem por esta abordagem, especialmente no setor em questão, os adversários demonstram que, na maior parte das vezes, preferem se defender de uma situação de ataque organizado, possivelmente apostando em sequências ofensivas estéreis (nas quais não ocorram ações que causem desequilíbrio defensivo e, por consequência, riscos à baliza) por parte da seleção da Espanha, a se sujeitarem a contra-ataques, durante os quais, em teoria, estarão em inferioridade numérica e/ou, experimentando crises de espaço em seu bloco defensivo ${ }^{29-30}$. Tais inferências corroboram os achados de Tenga et al. ${ }^{31}$, que indicaram que o método de jogo baseado em contra-ataques é mais efetivo contra blocos defensivos desequilibrados, enquanto o método baseado em ataque organizado é mais efetivo contra defesas equilibradas (pressão alta, com apoio e cobertura constantes).

Ainda no setor defensivo observou-se maior incidência de fragmentos constantes do jogo $(e=5,31)$ em comparação às outras FAR, o que permite uma análise importante sobre o padrão comportamental da equipe espanhola. Ao recuperar a posse de bola 
mais frequentemente através de lances de bola parada ou defesas do goleiro, a Espanha demonstra tendência a evitar o confronto direto $(1 \mathrm{x} 1)$ em seu setor defensivo. Tal inferência é possível ao se observar o valor residual significativamente menor de desarmes $(e=-4,57)$ nesta parte do campo. Estes resultados estão de acordo com as observaçóes de Turner e SAYERS $^{32}$, que também verificaram maior número de açôes de recuperaçáo da posse de bola através de desarme neste setor do campo. No presente estudo também verificou-se maior frequência de desarmes (e $=4,85)$ no setor médio defensivo, local que pode ser considerado crítico para recuperação da bola através de confronto direto dentro do modelo de jogo espanhol. Estes dados confirmam alguns dos objetivos gerais do jogo da Espanha durante a fase defensiva, que são os de impedir a criação de situaçôes de superioridade numérica ou posicional em espaços próximos à própria baliza e também o de evitar que os defensores sejam superados por desmarcaçóes do adversário, pelo controle de seus deslocamentos ${ }^{27}$.

Através dos resultados do presente estudo foi possível verificar importantes características do modelo de jogo da seleção de futebol da Espanha, no que diz respeito às variáveis relativas à transição defesa-ataque ${ }^{8}$. Assim, considerando a efetividade deste tipo de padrão comportamental transicional, deduz-se que treinamentos baseados em princípios que contribuam para os processos de adaptação e modelação de tal comportamento afiguram-se essenciais para que os jogadores percebam e incorporem a organizaçáo e a dinâmica do modelo de jogo da equipe, visando o aumento da qualidade das açóes individuais e da eficácia coletiva no jogo ${ }^{33-34}$.

Estes resultados permitem dizer que para às açôes intra-setoriais de recuperação da bola, houve maior ocorrência de açôes relativas aos fragmentos constantes do jogo, no setor defensivo, de interceptaçôes, no setor médio defensivo e médio ofensivo, e de fragmentos constantes do jogo, no setor ofensivo, o que sugere que a seleção espanhola tende a evitar confrontos $1 \times 1$ no setor defensivo, informação evidenciada pelo menor número de desarmes observados neste setor.

Ao se considerar as formas de aquisição/recuperação da bola (FAR), verifica-se que a maior ocorrência de interceptaçôes, desarmes e fragmentos constantes do jogo se deu no setor médio defensivo, o que atesta a importância estratégica deste setor do campo sobre a frequência destas açôes e, novamente, demonstra uma tendência comportamental por parte da seleção da Espanha no que diz respeito à recuperação da bola.

Para trabalhos futuros, recomenda-se a análise de outras variáveis relativas à fase ofensiva, para que seja possível estabelecer relaçóes entre padróes comportamentais da transiçáo defesa-ataque e os resultados das sequências ofensivas.

\begin{abstract}
Analysis of patterns of offensive of the Spanish National Soccer Team in the 2014 FIFA ${ }^{\circledR}$ World Cup

This paper aims to identify the patterns of offensive transition (defense-attack) of the Spain National Soccer Team during the 2010 FIFA $^{\circledR}$ World Cup through the analysis of frequency of the forms of ball acquisition/recovery (FAR) and the areas of the field where these actions took place. The sample comprises 895 offensive sequences performed during the 7 matches of the Spain National Team in the tournament. For data collection, it was performed observations of televised matches. Data were registered and quantified by Excel spreadsheets. Descriptive analysis (frequency and percentage) was performed for the variables "area" and "FAR". Chi-squared test $\left(\chi^{2}\right)$ was used to compare case distribution among the values of the analyzed variables with significance level of $p<0.05$. It was calculated the standardized residuals (e) of "FAR" according to the area of the field and also of the areas of the field according to "FAR". For statistical procedures, IBM SPSS v.20 was used. The variables "interception" and "constant match fragments" showed significant higher frequency than the other FAR in all areas of the field, whereas "interception" presented higher frequency value in defensive $(e=2.73)$, pre-defensive (e $=5.51)$ and pre-offensive $(e=2.41)$ areas, and the variable "tackle" had significant higher frequency in the defensive area $(e=4.85)$. It is concluded that there were higher frequency of constant match fragments in the defensive area, interceptions in the pre-defensive and pre-offensive areas and, once
\end{abstract}


more, of constant match fragments in the offensive area, what suggests that the Spain National Team shows predisposition to avoid 1v1 challenges in the defensive area.

KeY WoRds: Soccer; Tactics; Match analysis; Offensive transition.

\section{Referências}

1. Hughes M, Franks IM. The essentials of performance analysis: an introduction. New York: Routlege; 2008.

2. Silva PM, Castelo J, Santos P. Caracterização do processo de análise do jogo em clubes da 1a liga portuguesa profissional de futebol na época 2005/2006. Rev Bras Educ Fís Esporte. 2011;25:441-53.

3. Garganta J. Trends of tactical performance analysis in team sports: bridging the gap between research, training and competition. Rev Port Cien Desp. 2009;9:81-9.

4. Lago-Peńas C. The influence of match location, quality of opposition, and match status on possession strategies in professional association football. J Sports Sci. 2009;27:1463-9.

5. Duprat E, Caty D. Observation et classification des différents types de récupération du ballon lors de la phase défensive au football. eJRIEPS. 2007;12:34-50.

6. Amieiro N. Defesa à zona no futebol: um pretexto para reflectir sobre o “jogar”... bem, ganhando! 2a ed. Lisboa: Visão e Contextos; 2006.

7. Garganta J. Modelação táctica do jogo de futebol : estudo da organização da fase ofensiva em equipas de alto rendimento [tese]. Porto (PT): Universidade do Porto, Faculdade de Desporto; 1997.

8. Machado J, Barreira D, Garganta J. Attacking game-patterns in soccer: a sequential analysis of the World Cup 2010 winner team. In: Hughes M, Dancs H, Nagyváradi K, et al., editors. Research methods and performance analysis. Szombathely: University of West Hungary; 2010

9. Pollard R, Reep C, Hartley S. The quantitative comparison of playing styles in soccer. In: Reilly T, Lees A, Davids K, Murphy WJ, editors. Science and football: proceedings of the first World Congress of Science and Football. London: E. \& F.N. Spon; 1988. p.309-15.

10. Gréhaigne JF, Mahut B, Fernandez A. Qualitative observation tools to analyse soccer. Int J Perform Anal Sport. 2001; 1:52-61.

11. Barreira D, Garganta J, Guimarães P, Machado J, Anguera MT. Ball recovery patterns as a performance indicator in elite soccer. J Sports Eng Technol. 2014;228:61-72.

12. Machado JC, Barreira D, Garganta J. Eficácia ofensiva e variabilidade de padrôes de jogo em futebol. Rev Bras Educ Fís Esporte. 2013;27:667-77.

13. Lapresa D, Arana J, Anguera MT, Garzón B. Comparative analysis of sequentiality using SDIS-GSEQ and THEME: A concrete example in soccer. J Sports Sci. 2013;31:1687-95.

14. Sarmento H, Leitáo J, Anguera T, Campaniço J. Observational methodology in football: development of an instrument to study the offensive game in football. Motriz. 2009;5:19-24.

15. Añon IC, Lizana CJR, Calazans E, Machado JC, Teoldo I, Scaglia AJ. Performance da equipe do Barcelona e seus adversários nos jogos finais da Champions League e da Copa do Mundo de Clubes FIFA 2010. Rev Andal Med Deporte. 2014;7:13-20.

16. Safont-Tria BB, Nicolau JP, Traver VA, Riera JR. Propuesta de análisis de la táctica individual ofensiva en el fútbol. Apunts. 1996:63-71.

17. Kuper S, Szymanski S. Soccernomics. New York: Nation Books; 2009.

18. O’Donoghue P. Statistics for sport and exercise studies: an introduction. Oxon: Routledge; 2012.

19. Field A. Discovering statistics using IBM SPSS Statistics. 4th ed. London: Sage; 2013.

20. Cohen J. Quantitative methods in psychology. Psychol Bull. 1992;112:155-9.

21. Agresti A, Finlay B. Métodos estatísticos para as ciências sociais. 4a ed. Porto Alegre: Penso; 2012.

22. Teoldo I, Albuquerque M, Garganta J. Relative age effect in Brazilian soccer players: a historical analysis. Int J Perform Anal Sport. 2012;12:563-70.

23. Haberman SJ. The analysis of residuals in cross-classified tables. Biometrics. 1973;29:205-20.

24. Tabachnick B, Fidell L. Using multivariate statistics. 6th ed. London: Pearson Education; 2012. 
25. Landis R, Koch GG. The measurement of observer agreement for categorical data. Biometrics. 1977;33:159-74.

26. Barreira D, Garganta J, Anguera T. In search of nexus between attacking game-patterns, match status and type of ball recovery in European Soccer Championship 2008. In: Hughes M, Dancs H, Nagyváradi KP, et al., editors. Research methods and performance analysis. Szombathely: University of West Hungary; 2010

27. Moreno OPC. El modelo de juego del F.C. Barcelona. Pontevedra: MC Sports; 2010.

28. Pereni A, Di Cesare M. Zone play. Spring City: Reedswain, 1998.

29. Dugrand M. Football, de la transparence à la complexité. Paris: Presses Universitaires de France; 1989.

30. Mombaerts E. Pédagogie du football. Paris: Vigot, 1999.

31. Tenga A, Holme I, Ronglan LT, Bahr R. Effect of playing tactics on achieving score-box possessions in a random series of team possessions from Norwegian professional soccer matches. J Sports Sci. 2010;28:245-55.

32. Turner BJ, Sayers MGL. The influence of transition speed on event outcomes in a high performance football team. Int J Perform Anal Sport. 2010;10:207-20.

33. Worthington E. Learning \& teaching soccer skills. North Hollywood: Wilshire Book; 1974.

34. Teoldo I, Garganta J, Greco PJ, Mesquita I, Muller E. Relação entre a dimensão do campo de jogo e os comportamentos táticos do jogador de futebol. Rev Bras Educ Fís Esporte. 2011;25:79-96.

Rodrigo de Miranda Monteiro Santos Núcleo de Pesquisa e Estudos em Futebol Departamento de Educação Física Universidade Federal de Viçosa Av. P.H. Rolfs, s/n. 36570-90o - Viçosa - MG - BRASIL e-mail: mirandamonteiro@globo.com

Recebido para publicação: 30/05/2013

1a. Revisão: 16/07/13

2a. Revisão: 14/04/14

3a. Revisão: 30/04/14

Aceito: 20/08/2014 